\section{Sensitivity of Acute Flaccid Paralysis Surveillance in Nigeria (2006-2015)}

\section{Abstract}

Background: Nigeria has made tremendous progress towards polio eradication. The country was removed from the list of polio endemic countries by the World Health Organization (WHO) in September 2015. The last cases of wild poliovirus (WPV) and circulating vaccine derived poliovirus (cVDPV) from acute flaccid paralysis (AFP) cases had onset of paralysis in July 2014 and May 2015 respectively. The country has completed the phase I of laboratory containment of poliovirus activities, has achieved and maintained certification standard surveillance and is now in the process of ensuring adequate documentation preparatory to certification in 2017.

Methods: We conducted a retrospective review of AFP surveillance performance in Nigeria between 2006 and 2015 from the AFP database at the WHO Country Office. We also reviewed rapid surveillance assessment reports conducted in various states of the country within the reporting period to identify surveillance strengths and gaps as well as recommendations put forward to improve polio surveillance performance.

Results: The sensitivity of AFP surveillance in Nigeria increased consistently over the past 10 years. The number of confirmed and polio compatible cases has reduced significantly during the reporting period. AFP reporting sites have been prioritized for active surveillance and community informants have been engaged and cut across several key stakeholders in community health care delivery system.

Conclusion: The AFP surveillance performance in Nigeria during the reporting period demonstrated high level of sensitivity that can be relied upon to timely detect polio outbreak. Residual surveillance gaps at sub national levels however exist and must be closed to be able to identify remaining areas of poliovirus transmission should such exist as well as promptly detect possible cases of importation. Quality surveillance is also required for certification.

Keywords: Acute flaccid paralysis; Poliovirus; Surveillance

\author{
Abdullahi Walla Hamisu', \\ Ticha Muluh Johnson ${ }^{1}$, \\ Kehinde Craig ${ }^{1}$, Braka Fiona ${ }^{1}$, \\ Richard Banda ${ }^{1}$, \\ Sisay G Tegegne ${ }^{1}$, \\ Ajiboye Oyetunji ${ }^{1}$, \\ Emelife $\mathrm{Obi}^{2}$ and Sani Gwarzo ${ }^{3}$
}

1 World Health Organization, Country Representative Office, Abuja, Nigeria

2 National Primary Health Care Development Agency, Abuja, Nigeria

3 Federal Ministry of Health, Abuja, Nigeria

Corresponding author:

Abdullahi Walla Hamisu

\section{झabdullahih@who.int}

$\mathrm{MD}, \mathrm{MPH}$, National Surveillance Officer, World Health Organization, Nigeria Country Office, Abuja, Nigeria.

Tel: +2348036177515

Citation: Hamisu AW, Johnson TM, Craig K, et al. Sensitivity of Acute Flaccid Paralysis Surveillance in Nigeria (2006-2015). J Infec Dis Treat. 2016, 2:2.

Received: July 13, 2016; Accepted: August 03, 2016; Published: August 06, 2016

\section{Introduction}

In 1988, the World Health Assembly committed WHO to eradicating poliomyelitis; and since then, significant progress has been made as the incidence of poliomyelitis has declined by more than $99 \%$. The main strategies which brought about this success included improved routine immunization, supplemental mass immunization, adequate outbreak response capacity and effective surveillance [1].

Nigeria has recorded tremendous progress in polio eradication. The last cases of WPV and CVDPV from AFP cases had onset of paralysis in July 2014 and May 2015 respectively; and the country was removed from the list of polio endemic countries in September 2015 [2].

For polio eradication, AFP surveillance is needed to identify possible areas of poliovirus transmission or cases of importation. Surveillance is also critical for documenting the absence of poliovirus circulation for polio-free certification [3]. The indicator used to determine if surveillance is sufficiently sensitive to detect poliovirus transmission is the non-polio AFP rate [4]. A country's surveillance system should be sensitive enough to detect at least one case of AFP for every 1,00,000 children under the age of 15- 
even in the absence of polio [5]; and in the case of polio endemic countries, at least two cases of AFP for every 1,00,000 children under the age of 15 are required [6].

AFP surveillance is especially challenging because only one of every 200 polio virus infections results in clinically apparent paralytic disease. This underscores the importance of detecting and investigating all AFP cases, the most characteristic clinical condition associated with poliovirus infection among infants and children using a standard case definition [7-9]. It is however important to note that AFP is also caused by other conditions such as transverse myelitis, Guillain Barre Syndrome, metabolic neuropathies and toxins such as lead [10].

Having realized the importance of surveillance and its role in polio eradication, the revised national polio eradication emergency plan in Nigeria has as one of its main objective, ensuring that the highest quality AFP surveillance is achieved in all states [11].

\section{Methods}

\section{Study area and population}

The study areas were all the 36 states and the Federal Capital Territory (FCT) of Nigeria (Figure 1).

\section{Description of structure and responsibilities of the AFP surveillance system}

The AFP surveillance system is both health facility and community based. As at 2015, there is a network of 5,550 prioritized reporting sites (both public and private health facilities) and 46,978 community informants (comprising of Patent Medicine Vendors, traditional and spiritual healers, traditional bone setters, Traditional Birth Attendants, etc.) spread across the 9550 political wards, 36 states and the Federal Capital Territory (FCT). The proportion of wards with community informants and reporting sites was $96 \%$ and $60 \%$ respectively. Not all the wards have health facilities and not all the health facilities are functional.

The key surveillance focal points included the national epidemiologist and 37 state epidemiologists, a total of 801 Local Government Area (LGA) Disease Surveillance and Notification Officers (DSNOs) and 792 LGA DSNO assistants. In addition, WHO supports the surveillance structure with 148 cluster consultants, a total of 446 LGA facilitators and 1,371 Field Volunteers (FVs).

The health facility focal person in a reporting site as well as other health workers/clinicians detect and report AFP cases to the LGA DSNO who in turn has the responsibility of investigating the case including stool specimen collection and transportation to the national polio laboratory under reverse cold chain. The LGA DSNO is supported by his/her assistant and WHO LGA facilitator. Community informants refer AFP cases to the nearest reporting site or report cases directly to the LGA DSNO. Active surveillance at LGA level is conducted by LGA DSNO, his/her assistant, WHO LGA facilitators and field volunteers.

At the state level, the state epidemiologist and the state DSNO coordinate surveillance activities including organizing monthly LGA DSNO meetings, outbreak investigation, supervision, training and sensitization activities. The WHO cluster consultants support the state and LGA surveillance focal points, conduct active surveillance, verify reported AFP cases and conduct 60 day follow up inadequate AFP cases, confirmed poliovirus cases and cases with Sabin virus.

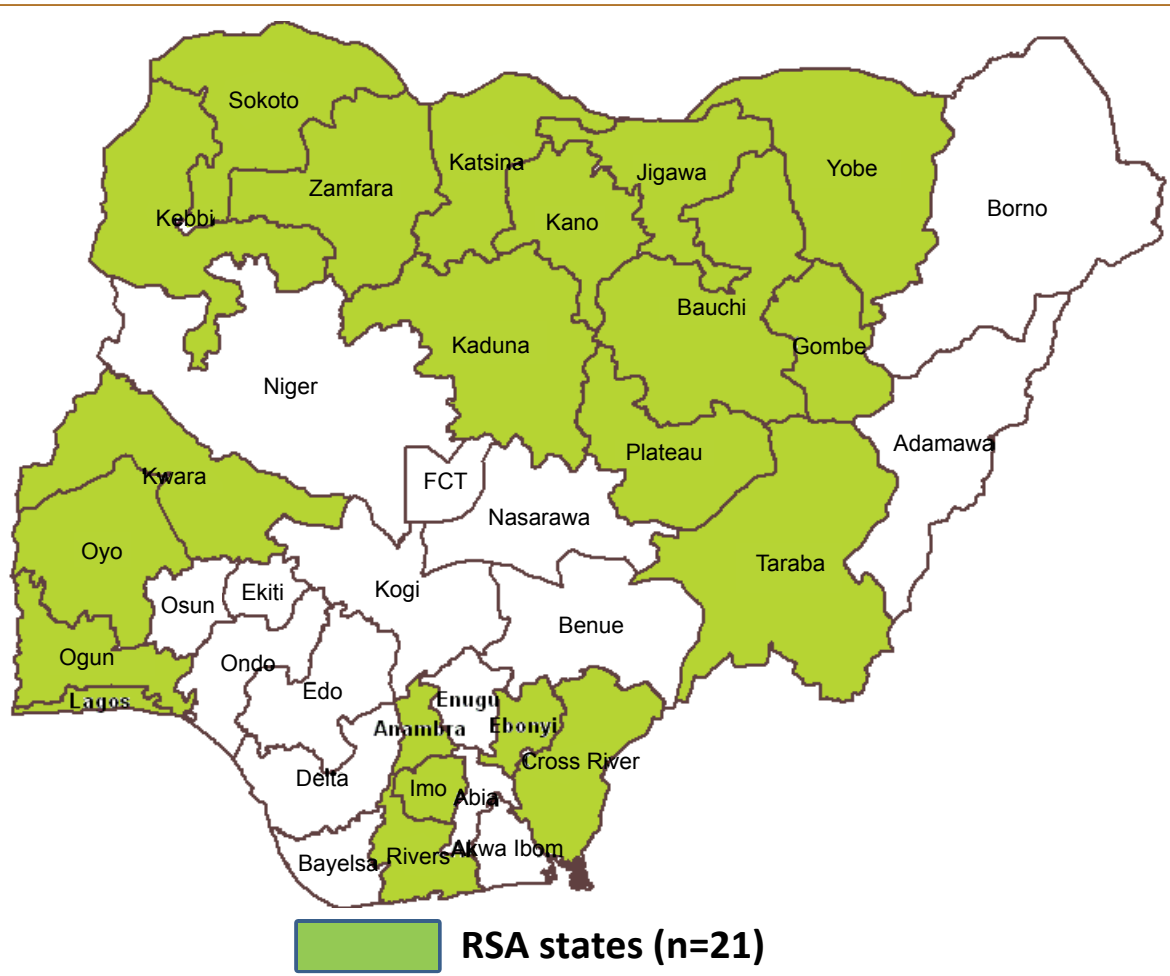

Figure 1 Map showing states in which rapid surveillance assessments were conducted (2011-2015). 
At the national level, surveillance is coordinated by the national epidemiologist who receives and analyses laboratory results from the two national polio laboratories and shares feedback with stakeholders, conducts supervision and monitors surveillance performance, organizes surveillance assessments and peer reviews, trainings and supports the polio laboratories and the national polio committees. WHO generally supports the surveillance system at all levels including provision of logistics support to the DSNOs, national polio laboratories and the national polio committees.

\section{Data collection and analysis}

A retrospective review of AFP surveillance performance in the country was conducted between 2006 and 2015. The two core AFP surveillance performance indicators of non-polio AFP and stool adequacy rates were analyzed using the AFP database at the WHO Country Office, Nigeria. We also reviewed the reports of rapid surveillance assessments conducted from across the country to identify surveillance strengths and gaps as well as recommendations put forward to improve polio surveillance performance.

\section{Results}

The sensitivity of AFP surveillance in the country has been on the increase in the past ten years. The years 2007 and 2015 recorded the least (5.9) and highest (18.8) levels of NP-AFP rates respectively; and these years also corresponded with the detection of least (4277) and highest (13948) numbers of AFP cases reported by the surveillance system (Tables 1-4).

The proportion of adequate stool specimens increased consistently from 2006 to 2015. The years 2006 and 2015 recorded the least (88\%) and highest (99\%) levels of stool adequacy rates respectively; and these years also corresponded with the detection of highest (1122) and least (0) numbers of WPV cases confirmed in the country.

The number of polio compatible cases has reduced significantly by $88 \%$, from 2006 when 193 compatible cases were classified to 2015 when 24 cases were classified.

The number of circulating Vaccine Derived Poliovirus cases confirmed reduced from the year (2009) with the highest number (154) recorded to 2015 when only one case was confirmed.

The proportion of high, medium and low reporting sites out of the total number (5550) of reporting sites was $80 \%, 20 \%$ and $10 \%$ respectively. The total number of community informants was 46978 . More than $80 \%$ of community informants consisted of Patent Medicine Vendors (PMVs), Traditional Birth Attendants (TBAs), Traditional Healers (THs), Community Leaders (CLs) and Traditional Bone Setters (TBSs).

Rapid surveillance assessments and peer reviews were conducted in 21 states from 2011 to 2015. Some high risk states (Kano, Kaduna, Kebbi, Sokoto, Katsina and Jigawa) had more than one of such surveillance assessments.
Table 1 Performance of Acute Flaccid Paralysis in Nigeria, 2006-2015.

\begin{tabular}{|c|c|c|c|}
\hline Year & No. AFP reported & NP-AFP rate & \% Stool adequacy \\
\hline 2006 & 5175 & 6.5 & 88 \\
\hline 2007 & 4277 & 5.9 & 94 \\
\hline 2008 & 5536 & 6.5 & 93 \\
\hline 2009 & 5501 & 7.1 & 95 \\
\hline 2010 & 5996 & 8.6 & 5 \\
\hline 2011 & 6099 & 8.5 & 9 \\
\hline 2012 & 7239 & 10.1 & 94 \\
\hline 2013 & 648 & 11.9 & 94 \\
\hline 2014 & 8 & 14.5 & 99 \\
\hline 2015 & 10506 & 18.8 & 98 \\
\hline
\end{tabular}

Table 2 Confirmed and Compatible Polio Cases in Nigeria, 2006-2015.

\begin{tabular}{|c|c|c|c|}
\hline Year & No. WPV & No. cVDPV & No. Compatible \\
\hline 2006 & 1122 & 21 & 193 \\
\hline 2007 & 285 & 68 & 74 \\
\hline 2008 & 798 & 65 & 93 \\
\hline 2009 & 388 & 154 & 76 \\
\hline 2010 & 21 & 27 & 79 \\
\hline 2011 & 62 & 33 & 140 \\
\hline 2012 & 122 & 8 & 67 \\
\hline 2013 & 53 & 4 & 81 \\
\hline 2014 & 6 & 30 & 35 \\
\hline 2015 & 0 & 1 & 24 \\
\hline
\end{tabular}

Table 3 Distribution of Community Informants by Type in Nigeria, 2015.

\begin{tabular}{|c|c|}
\hline Type & Number \\
\hline Patent Medicine Vendor & 14304 \\
\hline Traditional Birth Attendant & 10019 \\
\hline Traditional Healer & 7407 \\
\hline Spiritual Healer & 3100 \\
\hline Traditional Bone Setter & 4081 \\
\hline Community Leader & 4701 \\
\hline Others & 3366 \\
\hline Total & 46978 \\
\hline
\end{tabular}

Table 4 Distribution of Reporting Sites by Priority Level in Nigeria, 2015.

\begin{tabular}{|c|c|}
\hline Priority Level & Number \\
\hline High & 2090 \\
\hline Medium & 1847 \\
\hline Low & 1613 \\
\hline Total & $\mathbf{5 5 5 0}$ \\
\hline
\end{tabular}

\section{Discussion}

We found that the sensitivity of AFP surveillance in Nigeria has consistently increased over the past ten years and has exceeded the minimum WHO requirement. The highest level of performance was achieved in 2015. We believe that this high level of performance is attributable to enhanced active surveillance and the sheer number and spread of surveillance network of prioritized reporting sites and community informants. We also found that polio surveillance sensitivity was maintained 
at high level even in conflict areas of north eastern Nigeria affected by Boko Haram insurgency [12]. The main sources of AFP reporting in Nigeria were the AFP focal persons in reporting sites, other health workers/clinicians and informants [13]. The surveillance sensitivity was further enhanced by the introduction of environmental surveillance in the country from $2011[14,15]$.

AFP surveillance sensitivity in Nigeria is by far higher than in other countries such as Pakistan, Ghana, Zambia, South Africa, India and Australia [16-20]. This high performance notwithstanding, surveillance gaps still exist as exhibited by classification of polio compatible cases, confirmation of orphan viruses as well as findings from recent rapid surveillance assessments which included knowledge gaps among key surveillance personnel, missed AFP cases, inadequate active surveillance and documentation [21-24].

One of the key public health implications of our finding is that AFP surveillance indicators by themselves are not absolute in determining the true surveillance strengths. These indicators need to be supplemented with forms of monitoring activities such as rapid assessments, peer reviews and supervision. We found that adequate logistics support, training/sensitization, feedback and supportive supervision are what is required for surveillance to perform optimally.

One of the key limitations of this paper is the inadequate documentation of the trend of the number of reporting sites and community informants from 2006 to 2015 in the country. Thus the contribution of increasing number of reporting network to improving AFP surveillance sensitivity may be inadvertently lost. In addition, some of the surveillance assessments and peer reviews conducted during the reporting period have not been properly documented or published.

We recommend that surveillance gaps identified during rapid surveillance assessments be closed immediately considering that polio certification is around the corner. Documentation of all surveillance activities and archiving of information should be given priority along with active surveillance, training, sensitization and timely feedback to all stakeholders. Finally, surveillance in border, hard to reach and among other underserved populations such as migrants, nomadic and internally displaced persons should be enhanced to further ensure that poliovirus transmission or cases of importation are not missed. 


\section{References}

1 Abdel-Mannan OA, Harris MJ, Parker JA, Aly GS, El-Sayed NM (2010) Testing clinical surveillance of acute flaccid paralysis in Egypt posteradication of poliomyelitis. Tropical Medicine \& International Health 15: 1395-1400.

2 Morales M, Tangermann RH, Wassilak SGF (2016) Progress towards Polio Eradication - Worldwide, 2015-2016. Morbidity and mortality weekly report 65: 2015-2016.

3 Nundlall TR (2010) Global Situation of Poliomyelitis and Acute Flaccid Paralysis surveillance Global situation - 1988. WHO, pp: 1-45.

4 Budd J, Fowler B, Brown A, Swann S, Leung J, et al. (2013) Varicella Death of an Unvaccinated, Previously Healthy Adolescent-Ohio, 2009. Morbidity and Mortality Weekly Report 62: 261-263.

5 (2000) Rotarians Mobilizing for Surveillance what is Polio Surveillance? Frequently Asked Questions, pp: 1-8.

6 AFP Surveillance (2016) Clinical aspects 2016.

7 Gary HE, Sanders R, Pallansch MA (1997) A Theoretical Framework for Evaluating the Sensitivity of Surveillance for Detecting Wild Poliovirus: I. Factors Affecting Detection Sensitivity in a Person with Acute Flaccid Paralysis. Journal of Infectious Diseases, pp: 135-140.

8 Article O (2012) A Study of Various Factors Determining the Quality of Acute Flaccid Paralysis Surveillance of Beed District. pp: 30-37.

9 Core C (2012) Contributing towards Polio Eradication in Ethiopia Paper II. AFP case detection and status of surveillance in pastoralist and semi-pastoralist communities of CORE Group Polio Project implementation Districts (woredas) in Ethiopia.

10 April SM (2012) Nigeria's revised National Polio Emergency Plan (rNPEP) 2012 \& Progress so far.

11 Hamisu AW, Johnson TM, Craig K, Mkanda P, Banda R, et al. (2015) Strategies for Improving Polio Surveillance Performance in the Security-Challenged Nigerian States of Adamawa, Borno and Yobe during 2009-2014. Journal of Infectious Diseases, p: 530.

12 Bassey BE, Rui VG, Gasasira AN, Pascal M, Weldegbriel G, et al.
(2014) Characteristics of Acute Flaccid Paralysis Reported by the Surveillance System and Verified by WHO Officer in Akwa Ibom State-Nigeria, 2006-2012. Health 6: 2602.

13 Muluh TJ, Hamisu AW, Craig K, Mkanda P, Andrew E, et al. (2016) Contribution of Environmental Surveillance Toward Interruption of Poliovirus Transmission in Nigeria, 2012-2015. Journal of Infectious Diseases, p: 767.

14 States R, Weldegebriel G, Adeneji A, Gasasira A, Okello D, et al. (2015) Environmental Surveillance for Poliovirus in Polio. High 3: 655-663.

15 Faheem MU, Haroon MZ, Khan AA, Shaukat M, Anwar SA (2015) Acute Flaccid Paralysis Surveillance: A 5 Years Study Of Bannu, Pakistan. Journal of Ayub Medical College Abbottabad 27: 673-676.

16 Odoom JK, Afia N, Ntim A, Sarkodie B, Addo J, et al. (2014) Evaluation of AFP surveillance indicators in 14, pp: 1-8.

17 Length F (2014) Performance of acute flaccid paralysis surveillance system in Zambia: 2000 to 2009. Analysis of secondary data 3: 75-81.

18 Access $O$ (2013) Evaluating the acute flaccid paralysis surveillance system in South Africa, 2005-2009 - an analysis of secondary data. Pan African Medical Journal 8688: 2005-2009.

19 Singh J, Foster SO (2000) Original Articles 35: 311-315.

20 Watkins RE, Martin PAJ, Kelly H, Madin B, Watson C (2009) An evaluation of the sensitivity of acute flaccid paralysis surveillance for poliovirus infection in Australia. BMC infectious diseases 13: 1-13.

21 Emelife O, Weldegebriel G, Onuekwusi I, Gera R, Wolff C (2011) Review of the AFP surveillance system in Kano state, Nigeria.

22 June R (2011) Rapid Assessment of Acute Flaccid Paralysis (AFP) Surveillance in Kebbi State, Nigeria.

23 June R (2011) Rapid Assessment of Acute Flaccid Paralysis (AFP) Surveillance in Plateau State, Nigeria.

24 Isibor I, Gasasira A, Mkanda P, Weldegriebriel G, Bassey BE, et al. (2014) Rapid assessments of acute flaccid paralysis surveillance in seven key polio high risk states in Northern Nigeria. Pak J Med Sci 2: $33-40$. 